\title{
Inducible cardiomyocyte injury within the atrioventricular conduction system uncovers latent regenerative capacity in mice
}

\author{
Lin Wang, ${ }^{1}$ Minoti Bhakta, ${ }^{1}$ Antonio Fernandez-Perez, ${ }^{1}$ and Nikhil V. Munshi ${ }^{1,2,3,4}$ \\ 'Department of Internal Medicine (Cardiology Division), ${ }^{2}$ Department of Molecular Biology, ${ }^{3}$ McDermott Center for Human Growth and Development, and ${ }^{4}$ Center for Regenerative Science and Medicine, UT \\ Southwestern Medical Center, Dallas, Texas, USA.
}

\begin{abstract}
The cardiac conduction system (CCS) ensures regular contractile function, and injury to any of its components can cause cardiac dysrhythmia. Although all cardiomyocytes (CMs) originate from common progenitors, the CCS is composed of biologically distinct cell types with unique functional and developmental characteristics. In contrast to ventricular cardiomyocytes, which continue to proliferate after birth, most CCS cells terminally exit the cell cycle during fetal development. Although the CCS should thus provide a poor substrate for postnatal injury repair, its regenerative capacity remains untested. Here, we describe a genetic system for ablating CMs that reside within the atrioventricular conduction system (AVCS). Adult mouse AVCS ablation resulted in regenerative failure characterized by persistent atrioventricular conduction defects and contractile dysfunction. In contrast, AVCS injury in neonatal mice led to recovery in a subset of these mice, thus providing evidence for CCS plasticity. Furthermore, CM proliferation did not appear to completely account for the observed functional recovery, suggesting that mechanisms regulating recovery from dysrhythmia are likely to be distinct from cardiac regeneration associated with ventricular injury. Taken together, we anticipate that our results will motivate further mechanistic studies of CCS plasticity and enable the exploration of rhythm restoration as an alternative therapeutic strategy.
\end{abstract}

\section{Introduction}

The cardiac conduction system (CCS) ensures regular contractile function, and injury to any of its components can cause cardiac dysrhythmia. For example, atrioventricular node (AVN) injury can lead to complete heart block (CHB), a common clinical arrhythmia often requiring permanent pacemaker implantation. In contrast to device therapies, a major goal of regenerative medicine is to promote endogenous repair mechanisms for therapeutic benefit (1). Owing to sustained neonatal proliferative capacity (2), ventricular myocytes (VMs) (3) are capable of regeneration during this developmental window. Although CCS and working (i.e., atrial and ventricular) cardiomyocytes $(\mathrm{CMs})$ originate from cardiac progenitors $(4,5)$, CCS cells constitute a biologically distinct lineage with unique functional and developmental characteristics (6-14). Indeed, as opposed to neonatal VMs, the AVN undergoes early terminal differentiation, exits the cell cycle, and proliferates slowly (15-18), suggesting negligible regenerative capacity after birth $(3,19)$. Nevertheless, atrioventricular conduction system (AVCS) plasticity has not been directly evaluated, since genetic models for cell type-specific injury do not exist.

\section{Related Commentary: https://doi.org/10.1172/JCl152185}

Conflict of interest: The authors have declared that no conflict of interest exists. Copyright: @ 2021, American Society for Clinical Investigation.

Submitted: March 31, 2020; Accepted: August 5, 2021; Published: October 1, 2021

Reference information: J Clin Invest. 2021;131(19):e138637.

https://doi.org/10.1172/JCl138637.
Using a Cx30.2 enhancer directing expression to the AVCS (11), we established a genetic system for inducible AVCS injury (Figure 1A) that resulted in atrioventricular block (AVB) following tamoxifen-induced injury. Remarkably, we identified unexpected AVCS plasticity that was unique to injured neonatal mice and remained durable for up to 6 months. Interestingly, CM proliferation did not appear to entirely explain the recovery from dysrhythmia we observed in the injured neonatal mice. Taken together, our findings establish an inducible AVB (iAVB) mouse model, identify a latent AVCS regenerative capability, and hint at unique cellular mechanisms for AVCS plasticity.

\section{Results and Discussion}

Using a previously characterized AVCS-specific Cx30.2 enhancer(11), we created $\operatorname{Tg}(\mathrm{Cx} 30.2-\mathrm{MerCreMer})$ mice (Supplemental Figure 1A; supplemental material available online with this article; https://doi.org/10.1172/JCI138637DS1). We confirmed AVCS recombination in $\mathrm{Tg}(\mathrm{Cx} 30.2-\mathrm{MerCreMer})^{/+}$Rosa26 ${ }^{\text {lacz/+ }}$ mice following i.p. or s.c. tamoxifen administration (Supplemental Figure 1, B-H). In brief, we found that Cre recombination was leakproof, inducible, and largely confined to the AVCS (Supplemental Figure 1, B-H). Collectively, these experiments demonstrated that $\operatorname{Tg}(\mathrm{Cx} 30.2-$ MerCreMer) mediated efficient, tamoxifen-inducible recombination within the AVCS.

In order to establish a system for conditional ablation of AVCS cells, we generated $\mathrm{Tg}(\mathrm{Cx} 30.2-\mathrm{MerCreMer})^{/+} \mathrm{Rosa}^{26^{\mathrm{DTA} / \mathrm{LacZ}}}$ triple-heterozygous iAVB mice along with Rosa26 $6^{\text {DTA/LacZ }}$ littermate controls (Figure 1A). Although diphtheria toxin fragment A (DTA) 


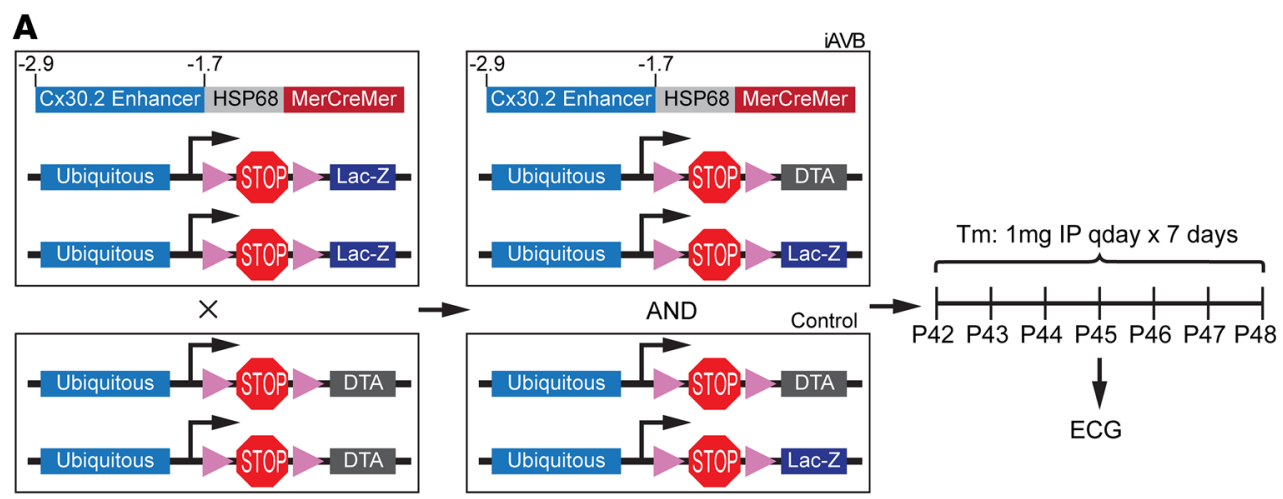

\section{B}
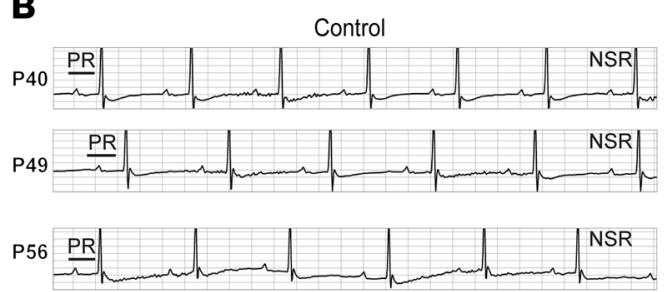

\section{C}
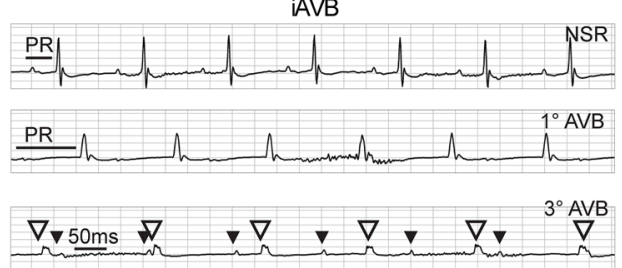

D

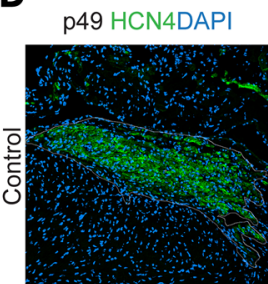

p55 HCN4DAPI
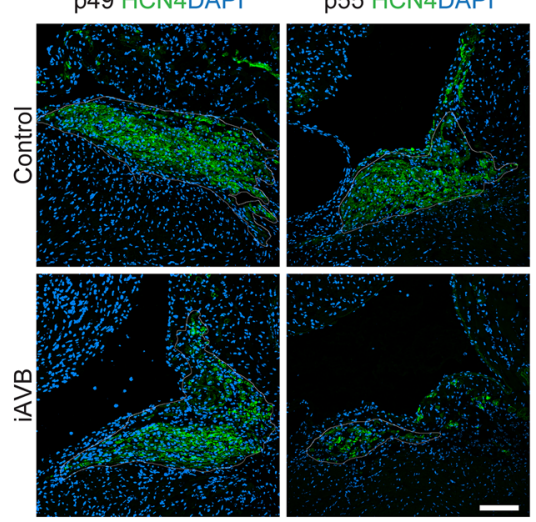

E

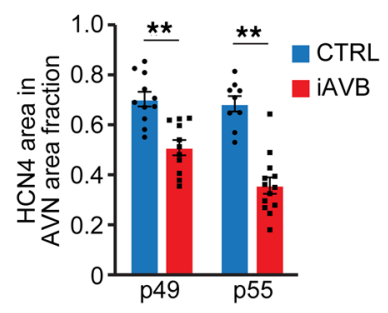

Figure 1. A genetic system for precise and inducible AVCS injury. (A) Experimental setup for tamoxifen-inducible AVCS injury. (B) Lead II ECG tracings at baseline (P40) and following tamoxifen administration (P49 and P56) in control mice. A representative tracing is shown $(n=12)$. (C) A similar analysis of iAVB mice showed NSR at baseline followed by progression from $1^{\circ}$ AVB (P-R prolongation) to $3^{\circ}$ AVB (AV dissociation) on P49 and P56, respectively. Black arrowheads designate $P$ waves (atrial depolarization); white arrowheads designate QRS complexes (ventricular depolarization). P-P and R-R intervals were fixed but distinct from one another. A representative tracing is shown $(n=16)$. (D) The hearts of iAVB and control (CTRL) mice were cryosectioned on P49 or P55 and stained for HCN4 (AVCS marker). A representative section is shown $(n=3)$. Scale bar: $100 \mu \mathrm{m}$. (E) The HCN4+ area was quantified as a fraction of the total AVN area $(n=3)$. Values represent the mean \pm SEM. ${ }^{* *} P<0.01$.

mice as a tool for controlled ablation of AVCS CMs.

Next, we evaluated the functional consequences of AVCS injury in adult iAVB mice (Figure 2A). We observed no significant difference in survival between adult iAVB and control mice (Figure 2B), despite clear differences in cardiac conduction (Figures 2, C and D, and Supplemental Figure 3). All adult iAVB mice developed irreversible $1^{\circ}$ or $3^{\circ}$ AVB (Figure 2E), suggesting a lack of regenerative capacity. Interestingly, we observed progressive LV dysfunction in iAVB mice with a reduction of approximately $15 \%$ in fractional shortening (FS) by 6 months of age compared with controls (Figure 2F), which is consistent with clinical studies showing that abnormal AV conduction is associated with cardiac contractile dysfunction (20, 21). Furthermore, left ventricular (LV) systolic dysfunction was proportionate to the degree of AVB (Figure 2G and Supplemental Figure 4) and not clearly attributable to LV fibrotic scar formation (Supplemental Figure 5). Moreover, we clearly observed progressive LV chamber dilation (Supplemental Figure 6, A and B) in adult iAVB mice, and careful inspection of histological images revealed evidence of patchy interstitial fibrosis (Supplemental Figure 6, C and D), consistent with changes observed following chronic right ventricular pac-

leakiness and nonspecific cardiac electrical effects were excluded by injecting control mice with tamoxifen (Figure 1B), iAVB mice had ECG results indicative of AVCS injury $\left(1^{\circ}\right.$ AVB on P49 and $3^{\circ}$ AVB on P56) after induction (Figure 1C). Histological analysis confirmed hyperpolarization-activated cyclic nucleotide-gated potassium channel 4-positive $\left(\mathrm{HCN}^{+}\right) \mathrm{CM}$ dropout and progressive fibrosis in iAVB mice (Figure 1, D and E, and Supplemental Figure 2, A and B), which are consistent with tamoxifen-inducible AVCS CM injury. Furthermore, we observed that iAVB/LacZ mice had less X-gal ${ }^{+}$AVCS cells than did control $\mathrm{Tg}(\mathrm{Cx} 30.2-\mathrm{MerCreMer})^{/+}$ Rosa26 ${ }^{\text {LacZ/+ }}$ (AVCS-iLacZ) mice on P50 and P180 (Supplemental Figure $2 \mathrm{C}$ ). Taken together, these experiments establish iAVB ing in patients $(22,23)$. In response to isoproterenol infusion, the $\mathrm{P}-\mathrm{P}$ and R-R intervals were shortened in adult iAVB mice, while atropine had minimal effect (Supplemental Figure 7). Collectively, these results demonstrate regenerative failure in adult iAVB mice, which had LV functional deterioration and a subset of pharmacological responses similar to those in patients with acquired AV conduction defects and long-term pacing (20).

Since regenerative capacity typically decreases with age, we reasoned that a younger AVCS would have a better chance of recovery. Therefore, we ablated AVCS cells in neonates by admin-

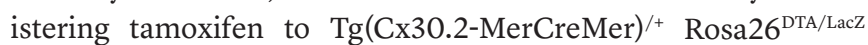
(iAVB) mice and Rosa26 $6^{\text {DTA/LacZ }}$ littermate controls on PO. Remark- 
A

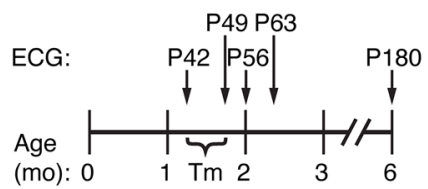

D

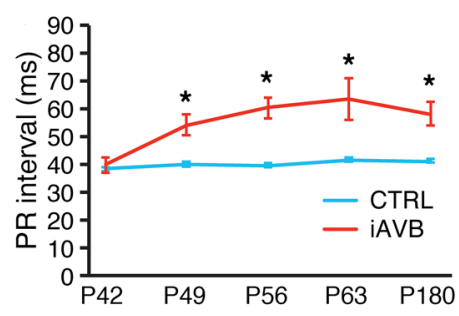

B

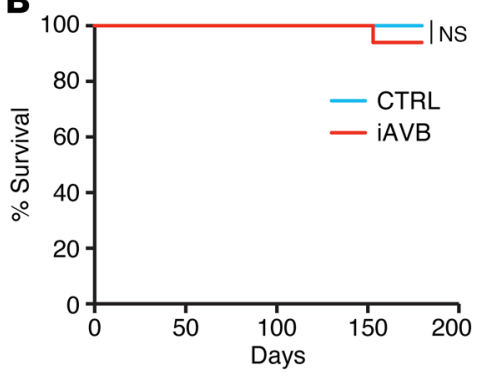

C

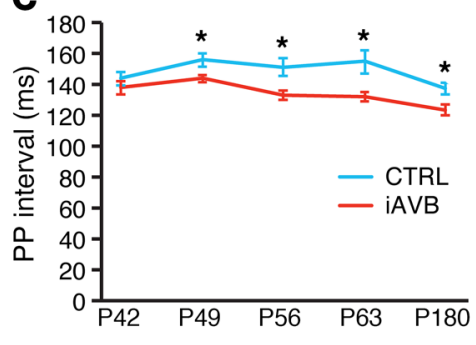

E

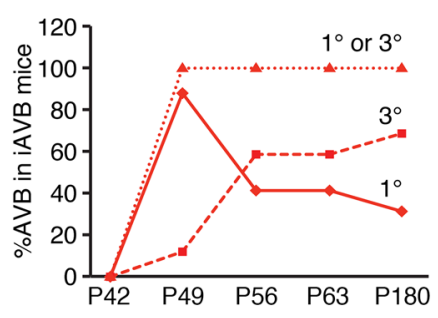

$\mathbf{F}$

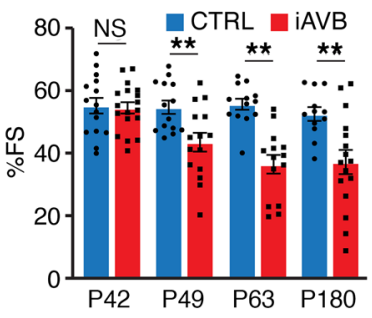

G

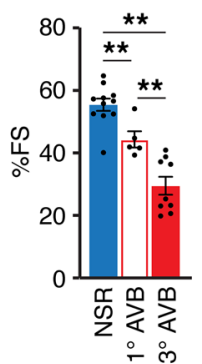

Figure 2. AVCS injury in adult mice results in regenerative failure and contractile dysfunction. (A) Schedule of ECG and echocardiogram acquisition for longitudinal analysis of adult control and iAVB mice. (B) Kaplan-Meier plot showing survival of iAVB versus control mice up to 6 months of age $(n=28)$. A log-rank statistical test was performed. (C) Serial analysis of P-P interval/sinus rate $(n=28)$. (D) Serial analysis of P-R interval/AV conduction times $(n=28)$. (E) Plot of the predominant rhythm versus time in iAVB mice $(n=28)$. (F) Serial analysis of FS in iAVB versus control mice. The numbers of mice analyzed at each time point were as follows: P42 (control, $n=15$; iAVB, $n=18$ ); P49 (control, $n=14$; iAVB, $n=15$ ); P63 (control, $n=13$; iAVB, $n=14$ ); P180 (control, $n=12$; iAVB, $n=16$ ). (C) Comparison of FS between mice with $1^{\circ}$ iAVB or $3^{\circ}$ iAVB and control mice with NSR on P63 (NSR mice, $n=13 ; 1^{\circ}$ iAVB mice, $n=5 ; 3^{\circ} \mathrm{i} A \mathrm{VB}$ mice, $n=9$ ). Comparisons in $\mathbf{G}$ remained significant after correction for multiple-hypothesis testing. Values represent the mean $\pm \mathrm{SEM}$. ${ }^{*} P<$ $0.05,{ }^{* *} P<0.01$ by 2 -tailed Student's $t$ test (C, $\mathbf{D}, \mathbf{F}$, and $\left.\mathbf{G}\right)$.

ably, although a littermate control remained in normal sinus rhythm (NSR) throughout the time course (Supplemental Figure $8 \mathrm{~A})$, we observed $1^{\circ}$ followed by $2^{\circ} \mathrm{AVB}$ with a return to NSR in an iAVB mouse (Supplemental Figure 8B). On the basis of this initial result, we performed serial ECGs on a larger cohort of neonatal, juvenile, and adult iAVB mice. Juvenile $(n=16)$ and adult $(n=17)$ iAVB mice showed no evidence of recovery, yet $40 \%(n=42)$ of the surviving neonatal iAVB mice (Supplemental Figure 9) recovered from dysrhythmia, consistent with a limited AVCS regenerative potential (Figure 3A). Given the observed spectrum of AVB among injured mice, we noted that mice with $1^{\circ}$ AVB had a $64 \%$ recovery rate, whereas those with $2^{\circ}$ or $3^{\circ} \mathrm{AVB}$ had a $29 \%$ recovery rate (Figure $3 \mathrm{~B})$. Interestingly, mice with $1^{\circ} \mathrm{AVB}$ recovered quickly, while those with a higher degree of AVB had slower recovery kinetics (Figures 3C and Supplemental Figure 10). Taken together, these results demonstrate a discrete AVCS regenerative capacity in neonatal mice that varied according to the extent of injury, similar to what has been observed in fetal $(24,25)$ and neonatal $(26)$ working myocyte injury models.

Next, we performed paired ECG and histological analyses of injured $\mathrm{iAVB} / \mathrm{LacZ}$ mice using X-gal staining to trace the fate of $\mathrm{LacZ}^{+}$cells in comparison with $\mathrm{Tg}(\mathrm{Cx} 30.2-\mathrm{MerCreMer})$ Rosa26 $6^{\mathrm{Lac} Z /+}$ (AVCS-iLacZ) control mice. On the basis of our observation of maximal injury between P10 and P17 in iAVB mice, we analyzed time points before (P4 and P7) and after (P21 and P35) peak injury. On $\mathrm{P} 4$, we found that $1^{\circ} \mathrm{AVB}$ correlated with loss of $\mathrm{X}-$ gal $^{+}$cells (Figures 3D and Supplemental Figure 11), consistent with AVCS injury. From P7 to P35, we observed increasing numbers of X-gal ${ }^{+}$AVCS cells, although they never reached the quantities observed in the uninjured mice. Taken together, these results document AVCS ablation and subsequent recovery at the cellular level in neonatal mice. In addition, the data suggest that recovery from dysrhythmia was mediated in part by preexisting $\mathrm{X}-$ gal $^{+}$AVCS cells (see also Supplemental Figure 13).

Although a subset of neonatal mice recovered from dysrhythmia 1 month after injury, the durability of this response remained unclear. To address this issue, we evaluated neonatal, juvenile, and adult iAVB mice at 6 months of age by ECG and histological analyses. Similar to injured adults (Figure 2, B and E), juvenile iAVB mice demonstrated normal survival and persistent AVB, although their dysrhythmia became fixed relatively soon after injury induction (Supplemental Figure 12). Consistent with our previous results (Figure 3), we found that the recovered neonatal iAVB mouse remained in NSR (Supplemental Figure 13A, right panels). In contrast, juvenile and adult iAVB mice showed persistent $3^{\circ}$ AVB (Supplemental Figure 13, B and C, right panels). Furthermore, the recovered neonatal iAVB mouse demonstrated less fibrosis and better structural preservation of the AVCS compared with juvenile and adult iAVB mice (Supplemental Figure 13, A-C, left panels). The architectural destruction and fibrotic replacement of the AVCS seen in juvenile and adult iAVB mice resembles the histological changes observed in human hearts with acquired CHB (27). Consistent with our short-term analysis of recovered neonatal iAVB mice (Figure 3D), we clearly observed X-gal ${ }^{+}$AVCS cells 6 months after injury (Supplemental Figure 13A). In contrast, we failed to detect any significant X-gal ${ }^{+}$AVCS cells in juvenile or 
A

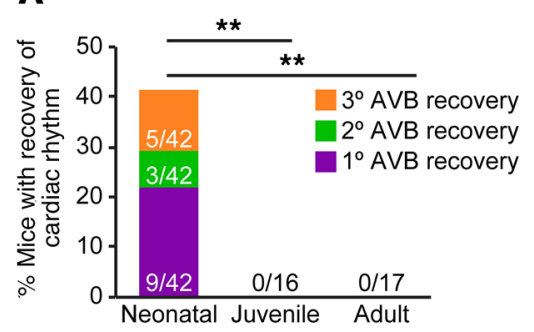

\section{B}

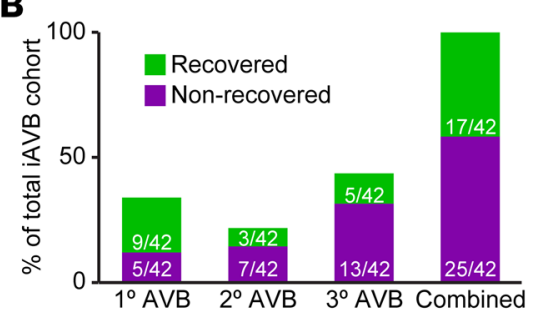

C

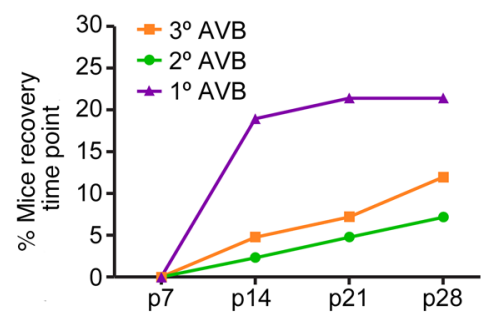

D

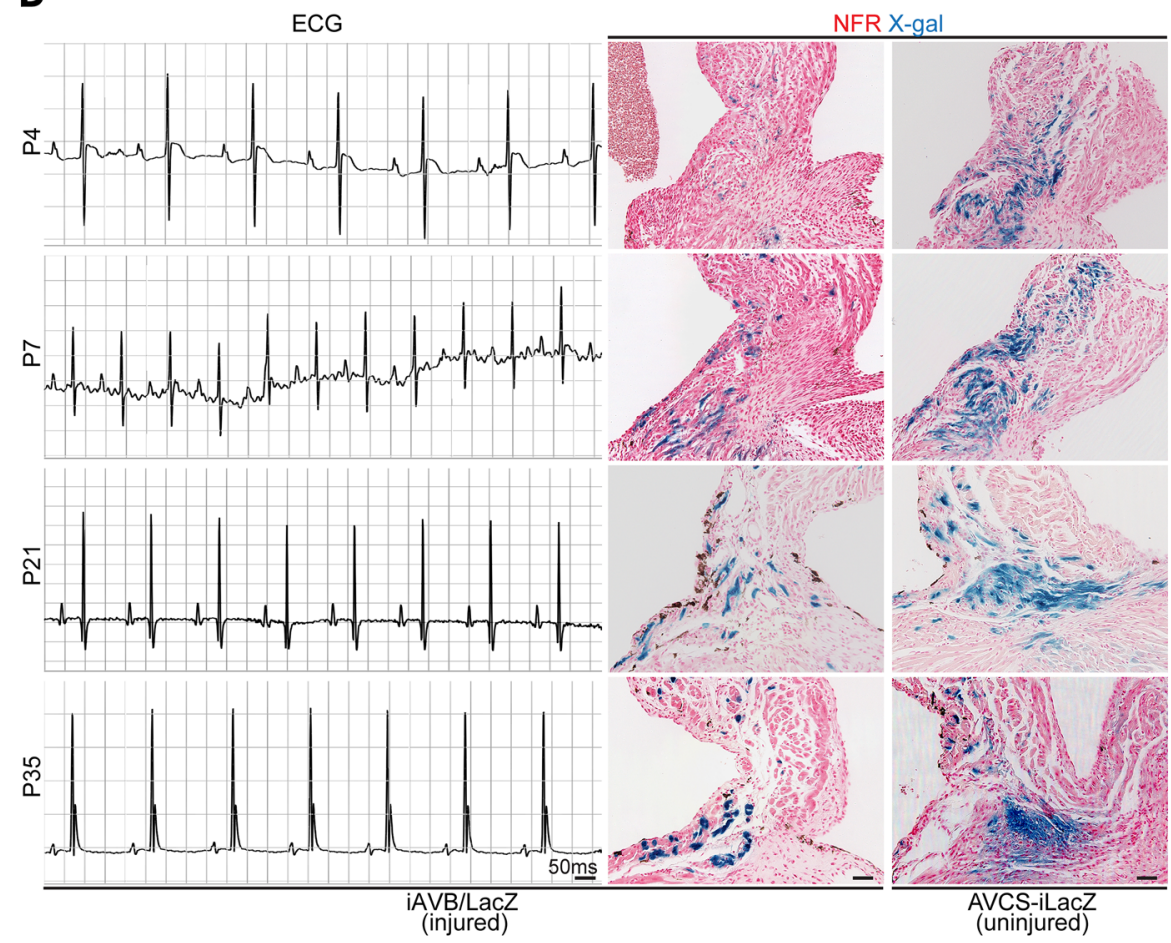

Figure 3. Neonatal AVCS plasticity contributes to recovery from dysrhythmia in a subset of mice. (A) Quantification of the percentage of animals showing ECG evidence of recovery under various AVCS injury paradigms (adult, $n=17$; juvenile, $n=16$; neonatal, $n=42$ ). ${ }^{* *} P<0.01$, by Fisher's exact test. (B) Relative distribution of the number of recovered versus nonrecovered animals with varying degrees of AVB $(n=42)$. (C) Plot of recovery from dysrhythmia as a function of time to demonstrate recovery kinetics in neonatal iAVB mice $(n=42)$. (D) ECG and histological analysis was performed on neonatal iAVB/ LacZ mice on P4, P7, P21, and P35. For comparison, the hearts of uninjured AVCS-iLacZ mice were harvested at identical time points following tamoxifen induction. X-gal staining was used to identify LacZ-expressing cells along with nuclear fast red (NFR) counterstaining $(n=3)$. Scale bars: $100 \mu \mathrm{m}$.

adult iAVB mice at 6 months of age (Supplemental Figure 13, B and C). Taken together, our results demonstrate sustained recovery from dysrhythmia in neonatal iAVB mice and suggest that AVCS regenerative failure in juvenile and adult mice is due in part to incomplete repopulation by preexisting AVCS CMs.

To address potential chronic effects of DTA in neonatal iAVB mice, we generated an independent cohort of mice injured on PO and examined at 6 months of age. By chance, a majority of the mice in this cohort developed $3^{\circ}$ AVB by 6 months of age despite consistent tamoxifen dosing regimens. Compared with

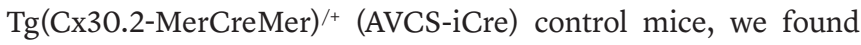
that FS was significantly reduced in iAVB mice (Supplemental Figure 14). Interestingly, neonatal iAVB mice with $1^{\circ} \mathrm{AVB}(n=2)$ had an FS of $41 \%$ compared with $26 \%$ in the remainder of the cohort. Consistent with the data for adult iAVB mice (Figure 2G), cardiac dysfunction appeared proportionate to injury severity, although the numbers of neonatal iAVB mice analyzed were limited. To exclude the possibility that transgenic Cre expression has significant phenotypic consequences, we also assessed FS and P-R intervals in AVCS-iCre mice compared with Rosa26 ${ }^{\text {DTA/LacZ }}$ controls on P28 and P180 following tamoxifen injection on PO. We found no significant difference in FS or P-R intervals at either time point (Supplemental Figure 15, A and B). Furthermore, we did not observe significant rates of AVB in AVCS-iCre mice following tamoxifen-induced injury on PO compared with iAVB mice (Supplemental Figure 15C). Taken together, these studies demonstrate the chronic effects of neonatal AVCS ablation and exclude Cre toxicity as a potential confounder of our phenotypic analysis.

Previous reports clearly document that proliferation of preexisting CMs accounts for neonatal VM regenerative capacity (3). However, our studies using iAVB neonatal mice (Figure 3D) suggest that $\mathrm{Cx} 30.2^{+} \mathrm{CMs}$ may not completely account for AVCS plasticity. Therefore, we conducted a time course analysis to investigate the cellular dynamics of neonatal AVCS recovery without lineage tracing (Supplemental Figure 16). We used phosphorylated histone $\mathrm{H} 3$ (p-H3) antibody to stain cells in mitosis, while $\alpha$-actinin and HCN4 marked all CMs and conduction CMs, respectively. We administered tamoxifen on PO to control (Rosa26 $\left.6^{\text {DTA/lacZ }}\right)$ and iAVB ( $\mathrm{Tg}(\mathrm{Cx} 30.2-\mathrm{MerCreMer})^{/+}$ Rosa26 ${ }^{\text {DTA/lacZ) }}$ mice and collected AVCS sections at multiple time points after ablation. We found that injury led to a statistically significant increase in overall cellular proliferation on $\mathrm{P} 4$ and P21 (Figure 4A). Interestingly, we did not observe significant proliferation of CMs within the AVCS in injured animals compared with controls (Figure 4B). In contrast, the proliferation of $\alpha$-actinin ${ }^{-}$(non-CM) cells increased significantly in injured mice 
A

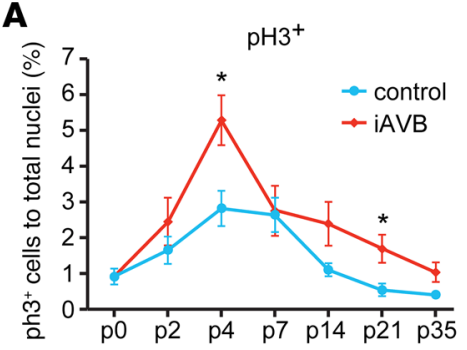

B

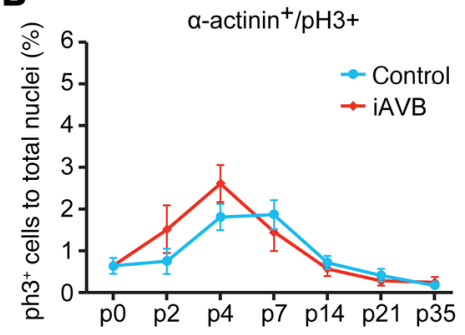

D

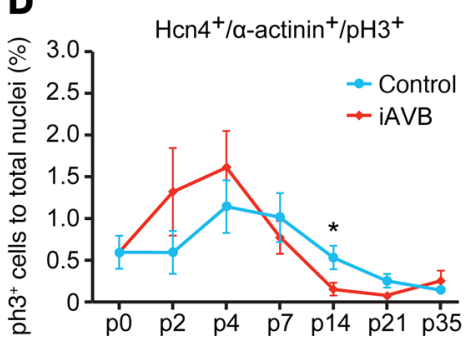

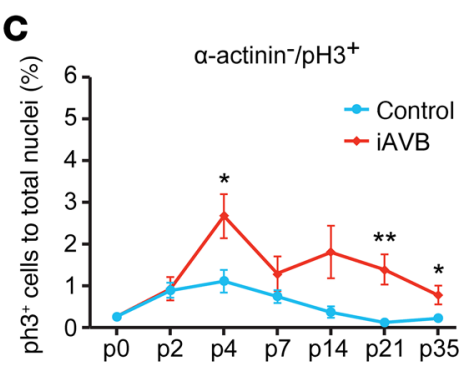

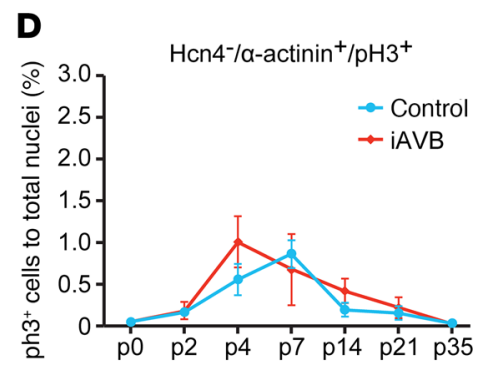

Figure 4. Cellular dynamics of proliferation following neonatal AVCS injury. (A-E) Cellular proliferation was quantified in the AVCS of control and iAVB mice. Each section was stained for $\mathrm{p}-\mathrm{H} 3$ (mitosis), $\alpha$-actinin (CMs), HCN4 (AVCS CMs), and DAPI (nuclei). Tamoxifen-induced injury was performed on $\mathrm{PO}$, and hearts were collected on P2, P4, P7, P14, P21, and P35. Comparisons between control and injured mice were made for (A) all proliferative cells $\left(\mathrm{p}-\mathrm{H}^{+}\right)$; (B) proliferative $\mathrm{CMs}\left(\mathrm{p}-\mathrm{H} 3^{+} / \alpha\right.$-actinin $\left.{ }^{+}\right)$; (C) proliferative non-CMs ( $\mathrm{p}-\mathrm{H3}^{+} / \alpha$-actinin $\left.{ }^{-}\right)$; (D) proliferative AVCS CMs $\left(\mathrm{p}-\mathrm{H}^{+} / \alpha\right.$-actinin $\left.{ }^{+} / \mathrm{HCN}^{+}\right)$; and (E) proliferative non-AVCS CMs $\left(\mathrm{p}-\mathrm{H}^{+} / \alpha\right.$-actinin ${ }^{+} /$ HCN4 $) . n=3 .{ }^{*} P<0.05$ and ${ }^{* *} P<0.01$, by 2 -tailed Student's $t$ test.

on P4, P21, and P35 (Figure 4C). To explore the possibility that the heterogeneity of mixed $\mathrm{HCN}^{+}$and $\mathrm{HCN}^{-}{ }^{-} \mathrm{AVCS} \mathrm{CMs}$ may obscure a biologically important signal, we also counted each cell population separately (Figure 4, D and E). Although there was a trend toward increased proliferation of $\mathrm{HCN}^{+} / \alpha$-actinin ${ }^{+}$ myocytes on P2 and P4 in the injured mice, none of the early differences reached statistical significance.

Given these findings, we next assessed the relative balance between cell death and proliferation at early time points in neonatal iAVB mice. We performed activated caspase-3 AVCS CM immunostaining on P2, P4, and P7 in control and AAVB mice (Supplemental Figure 17, A-D). Interestingly, we found that the percentage of apoptotic cells $(\sim 1.5 \%)$ was less than the percentage of proliferating cells $(-2.5-5 \%)$ in the AVCS at equivalent time points, suggesting that proliferation was favored over cell death in neonatal iAVB mice. Furthermore, the percentage of apoptotic cells in neonatal iAVB mice was intermediate between adult and juvenile iAVB mice (Supplemental Figure 17, A-D). Together with the fact that neonatal iAVB mice were able to recover from varying degrees of injury (Figure $3, \mathrm{~A}-\mathrm{C}$ ), we interpreted these findings to indicate that neonates had a unique ability to recover from AVCS injury rather than a relative resistance to DTA toxicity. Despite the recovery of iAVB mice, we found that the number of $\mathrm{HCN}^{+}$AVCS CMs diminished - yet increased in control mice - between P2 and P7 (Supplemental Figure 17E). Collectively, these results demonstrate prominent non-CM proliferation after neonatal AVCS injury. Furthermore, our data suggest the intriguing possibility that the neonatal AVCS deploys alternative mechanisms to achieve recovery from dysrhythmia in addition to proliferation of preexisting CMs.

In this report, we generated a genetic system for inducible AVCS injury. Using this paradigm, we show that AVCS ablation in adult mice resulted in persistent AV conduction block and impaired LV systolic function. Unexpectedly, we found that neonatal AVCS ablation uncovered a latent and durable regenerative capacity. In addition, we show that AVCS regeneration occurred in part by repopulation with preexisting AVCS CMs, although additional cellular contributions appeared to exist. Collectively, our data demonstrate that the neonatal AVCS harbored a heretofore unappreciated degree of plasticity and suggest that unique cellular mechanisms were functional, potentially including the remodeling of residual AVCS CMs and non-CMs. Given the clear role of gap junctions in CCS impulse propagation (28), we investigated $\mathrm{Cx} 30.2$, $\mathrm{Cx} 40$, and $\mathrm{Cx} 45$ distribution in recovered neonatal iAVB mice (Supplemental Figure 18). Remarkably, we observed a specific, $\mathrm{Cx} 40$-mediated remodeling event in non-CMs associated with electrical recovery. In parallel, we assessed for latent bypass tracts that could serve as alternative AV conduction pathways in iAVB mice. However, we failed to detect evidence of aberrant AV connections by pharmacological challenge (Supplemental Figures 19 and 20) or histological analysis (Supplemental Figures 21 and 22).

AVCS CM proliferation does not appear significantly increased following injury (Figure 4B), yet $\mathrm{X}^{-\mathrm{gal}^{+}}$cells were noted to increase (Figure 3D). How can we reconcile these observations? Although the differences in CM proliferation did not meet statistical significance, the number of proliferating AVCS CMs was numerically higher in iAVB mice on P2 and P4, so our analysis could be underpowered to detect a biologically meaningful difference. Alternatively, the fact that the proliferation of AVCS non-CMs was robust following injury (Figure 4C) suggests that AVCS CM proliferation is not the only mechanism for electrical recovery. One potential explanation for these seemingly discrepant observations is that non-CMs could proliferate and fuse with preexisting $\mathrm{X}$-gal ${ }^{+} \mathrm{CMs}$, as $\mathrm{CM}$ fusion has recently been recognized to regulate cardiac development and $\mathrm{CM}$ renewal (29-32). Another potential explanation is that non-AVCS X-gal ${ }^{+}$ $\mathrm{CMs}$, such as transitional atrial-nodal or nodo-ventricular CMs (33), could migrate into the injury zone and contribute to AVCS repair. Future work will focus on clarifying the molecular mech- 
anisms that endow the AVCS with inherent plasticity and designing new approaches to "unlock" this latent capacity for recovery from dysrhythmia. We anticipate that a detailed exploration of CCS plasticity will facilitate much-needed alternative therapeutic strategies for cardiac dysrhythmias.

\section{Methods}

See the Supplemental Methods for a detailed description of all experimental procedures.

Study approval. All animal procedures were approved by the IACUC of UT Southwestern Medical Center.

\section{Author contributions}

NVM conceptualized the study. LW, MB, and NVM collected data. LW, AFP, and NVM analyzed the data. NVM wrote the manuscript with input from LW and MB.

\section{Acknowledgments}

We thank the UT Southwestern Medical Center Histology Core Facility for technical expertise, Jose Cabrera for graphical assistance, Cheryl Nolen for mouse colony maintenance, Colby Ayers for statistical advice, Hesham Sadek, Eric Olson, and Ning Liu for critical review of the manuscript, and members of the Munshi laboratory for scientific discussions. We also thank Ari Bennett and Gabriela Orquera-Tornakian for key contributions during the revision process. This work was supported by the NIH (HL136604, HL133642, and HL135217, to NVM), the Burroughs Wellcome Fund (1009838, to NVM), and the March of Dimes Foundation (5-FY13-203, to NVM).

Address correspondence to: Nikhil V. Munshi, 5323 Harry Hines Blvd, Dallas, Texas 75390-8573, USA. Phone: 214.648.4001; Email:nikhil.munshi@utsouthwestern.edu.
1. Uygur A, Lee RT. Mechanisms of cardiac regeneration. Dev Cell. 2016;36(4):362-374.

2. Kotton DN, Morrisey EE. Lung regeneration: mechanisms, applications and emerging stem cell populations. Nat Med. 2014;20(8):822-832.

3. Porrello ER, et al. Transient regenerative potential of the neonatal mouse heart. Science. 2011;331(6020):1078-1080.

4. Cheng G, et al. Development of the cardiac conduction system involves recruitment within a multipotent cardiomyogenic lineage. Development. 1999;126(22):5041-5049.

5. Gourdie RG, et al. Terminal diversification of the myocyte lineage generates Purkinje fibers of the cardiac conduction system. Development. 1995;121(5):1423-1431.

6. Aanhaanen WT, et al. The Tbx2+ primary myocardium of the atrioventricular canal forms the atrioventricular node and the base of the left ventricle. Circ Res. 2009;104(11):1267-1274.

7. Bressan M, et al. Early mesodermal cues assign avian cardiac pacemaker fate potential in a tertiary heart field. Science. 2013;340(6133):744-748.

8. Espinoza-Lewis RA, et al. Shox 2 is essential for the differentiation of cardiac pacemaker cells by repressing Nkx2-5. Dev Biol. 2009;327(2):376-385.

9. Harris JP, et al. MyoR modulates cardiac conduction by repressing Gata4. Mol Cell Biol. 2015;35(4):649-661.

10. Liang X, et al. Transcription factor ISL1 is essential for pacemaker development and function. JClin Invest. 2015;125(8):3256-3268.

11. Munshi NV, et al. Cx30.2 enhancer analysis identifies Gata4 as a novel regulator of atrioventricular delay. Development. 2009;136(15):2665-2674.

12. Vedantham $V$, et al. RNA sequencing of mouse sinoatrial node reveals an upstream regulatory role for Islet- 1 in cardiac pacemaker cells. Circ
Res. 2015;116(5):797-803.

13. Wang J, et al. Pitx2 prevents susceptibility to atrial arrhythmias by inhibiting left-sided pacemaker specification. Proc Natl Acad SciUS A. 2010;107(21):9753-9758.

14. Zhang SS, et al. Iroquois homeobox gene 3 establishes fast conduction in the cardiac His-Purkinje network. Proc Natl Acad Sci U S A. 2011;108(33):13576-13581.

15. Sedmera D, et al. Spatiotemporal pattern of commitment to slowed proliferation in the embryonic mouse heart indicates progressive differentiation of the cardiac conduction system. Anat Rec A Discov Mol Cell Evol Biol. 2003;274(1):773-777.

16. Bakker ML, et al. Transcription factor Tbx3 is required for the specification of the atrioventricular conduction system. Circ Res. 2008;102(11):1340-1349.

17. Moskowitz IP, et al. A molecular pathway including Id2, Tbx5, and Nkx2-5 required for cardiac conduction system development. Cell. 2007;129(7):1365-1376.

18. Miquerol L, et al. Biphasic development of the mammalian ventricular conduction system. Circ Res. 2010;107(1):153-161.

19. Soonpaa MH, et al. Cardiomyocyte DNA synthesis and binucleation during murine development. Am J Physiol. 1996;271(5 pt 2):H2183-H2189.

20. De Sisti A, et al. Adverse effects of long-term right ventricular apical pacing and identification of patients at risk of atrial fibrillation and heart failure. Pacing Clin Electrophysiol. 2012;35(8):1035-1043.

21. Gillis AM, et al. HRS/ACCF expert consensus statement on pacemaker device and mode selection. J Am Coll Cardiol. 2012;60(7):682-703.

22. Adomian GE, Beazell J. Myofibrillar disarray produced in normal hearts by chronic electrical pacing. Am Heart J. 1986;112(1):79-83.

23. Karpawich PP, et al. Altered cardiac histology following apical right ventricular pacing in patients with congenital atrioventricular block. Pacing Clin Electrophysiol. 1999;22(9):1372-1377.

24. Sturzu AC, et al. Fetal mammalian heart generates a robust compensatory response to cell loss. Circulation. 2015;132(2):109-121.

25. Drenckhahn JD, et al. Compensatory growth of healthy cardiac cells in the presence of diseased cells restores tissue homeostasis during heart development. Dev Cell. 2008;15(4):521-533.

26. Darehzereshki A, et al. Differential regenerative capacity of neonatal mouse hearts after cryoinjury. Dev Biol. 2015;399(1):91-99.

27. Barra SN, et al. A review on advanced atrioventricular block in young or middle-aged adults. Pacing Clin Electrophysiol. 2012;35(11):1395-405.

28. Mezzano V, et al. Cell junctions in the specialized conduction system of the heart. Cell Commun Adhes. 2014;21(3):149-159.

29. Sawamiphak S, et al. Transient cardiomyocyte fusion regulates cardiac development in zebrafish. Nat Commun. 2017;8(1):1525.

30. Matsuura K, et al. Cardiomyocytes fuse with surrounding noncardiomyocytes and reenter the cell cycle. J Cell Biol. 2004;167(2):351-363.

31. Min YL, et al. Identification of a multipotent Twist2-expressing cell population in the adult heart. Proc Natl Acad Sci U S A. 2018;115(36):E8430-E8439.

32. Yellamilli A, et al. Abcg2-expressing side population cells contribute to cardiomyocyte renewal through fusion. FASEB J. 2020;34(4):5642-5657.

33. Bhattacharyya S, et al. Using Gjd3-CreEGFP mice to examine atrioventricular node morphology and composition. Sci Rep. 2019;9(1):2106. 